\title{
Optical Modeling of Laser Ablation for Microstructure Fabrication
}

\author{
M C Gower, E Davies, A S Holmes \\ Department of Electrical \& Electronic Engineering \\ Imperial College London \\ London SW7 2AZ, United Kingdom \\ E-mail:m.gower@imperial.ac.uk
}

\begin{abstract}
From just an a priori knowledge of the optical parameters of a laser beam, the delivery system together with a substrate's material properties, a ray-tracing model capable of predicting the 3-D topology of micro/nanostructures machined by pulsed laser ablation has been developed. The model includes secondary illumination effects produced by the microstructure created by successive pulses (wall reflections, refraction, wave guiding, shadowing, etc.) as well as the complete optical properties of the laser beam and delivery system. We have used material ablation by pulsed excimer lasers and associated beam delivery systems to demonstrate some of the capabilities of the model.
\end{abstract}

DOI:10.2961/jlmn.2013.01.0012

Keywords: Pulsed laser ablation, laser microstructuring, excimer laser micromachining

\section{Introduction}

Early work using lasers to fabricate micron sized features can be traced back to early 1982 when first reports appeared of using deep-UV excimer lasers to dry etch polymers by material ablation [1], a process soon thereafter demonstrated to have submicron spatial resolution [2,3] and the capability for microstructuring non-planar three-dimensional (3-D) shaped objects [4]. From these early beginnings, microprocessing materials with lasers has become widely regarded by manufacturing industry to be attractive because of its inherent flexibility for accommodating complex designs in a wide range of materials - from polymers, ceramics, glasses and crystals to metals, alloys and semiconductors. Ablation with pulsed solid state, $\mathrm{CO}_{2}$ and excimer lasers is now commonly used for drilling microholes - in circuit boards, interconnect packages and ink jet printer nozzles; repairing microelectronic and display devices; scribing and sectioning photovoltaic and photoemissive devices (wafers, cells and panels); prototyping MEMS devices; manufacturing medical devices and corneal sculpting in corrective refractive eye surgery.

There have been many theoretical approaches made to explain the material removal process by pulsed laser ablation. Excimer laser ablation of polymers in particular has perhaps received the widest attention $[5,6]$. The simplest approach used for this assumes a bond-breaking mechanism whereby repulsive electronic states are directly excited by photons absorbed in a substrate according to Beer's law. An incident laser fluence threshold $\mathrm{E}_{\mathrm{T}}$ for material removal arises as a result of a minimum density of bonds to be broken in order to overcome a material's enthalpy. This 'photochemical' model appears to work well for substrates irradiated at low fluences in the regime $\$ 20 \mathrm{x}$ the ablation threshold and at wavelengths for which there is a relatively large absorption coefficient $\left(\gtrsim 10^{5} \mathrm{~cm}^{-1}\right)$. At higher fluences and for materials less strongly absorbing 'photothermal' approaches have been developed whereby phonons, created by the relaxation to the ground state of photonexcited bound electronic states, cause heating and thermal decomposition of the material. In this case the ablation threshold arises as a result of the product formation rate needing to exceed the activation energy of the hot material. In intermediate regimes of fluence and absorption, attempts have been made to combine both photochemical and photothermal models to predict a total etch depth that is the summation of both [7]. Models have also been developed aimed at predicting the 3-D topology of ablated microstructures. Free-space angular spectrum propagation has been used $[8,9,10]$ to simulate the illumination incident on a sample from a mask imaging system, with an empirical etch function being used to estimate the consequent material removal. While this model was relatively successful at predicting wall angles of ablated holes and trenches $\geq 25 \mu \mathrm{m}$ in size, it did not account for secondary illumination effects produced by the microstructure created by successive pulses (wall reflections, refraction, wave guiding, shadowing, material interfaces in the substrate, etc), all of which become increasingly important as feature sizes are reduced. Ray tracing algorithms using 2-D pixel arrays to model the topology of ablated structures have been successful at predicting the shapes of relatively large $(>10 \mu \mathrm{m})$ hole-like features [ 11 ]. However assumptions made of collimated illumination are unrealistic when machining complex shapes with micron-sized structures produced by high numerical aperture mask imaging or focusing systems. To do so requires an intimate knowledge of (a) the properties of the laser beam; (b) the beam delivery system - optical components, aberrations, vignetting, mask features, numerical apertures, beam homogeneity, etc; (c) the impact the topology of the microstructure being machined has on its illumination by subsequent pulses. 
We report here on the development and experimental validation of a numerical model capable of predicting the topology of microstructures produced by laser ablation that include all the optical effects mentioned above. From only an a priori knowledge of the optical parameters of the laser beam, the beam delivery system together with the substrate material refractive and absorptive properties, the geometric shape of the micro/nanostructures machined by pulsed laser ablation can be predicted. These calculations can include properties of (a) the laser beam in terms of its wavelength, dimensions, pulse energy, number of pulses, divergence, polarization, spatial and temporal coherence; (b) the beam delivery system in terms of beam shaping, homogeneity, numerical aperture (NA), vignetting, optical aberrations, image ghosts, interference effects, off-axis mask illumination, amplitude and phase transmission characteristics of a mask; (c) the structure being created in the substrate by successive pulses in terms of geometric wall and floor reflections, light guiding, shadowing, substrate refraction, reflections from material interfaces.

Validation of our ablation model was carried out by comparing computations of the ablation rate and taper angle in polyimide with measurements made by ourselves and other workers $[11,12,13,14,15,16,17]$. Polyimide is important industrially as an electronic material, and for fabricating microvias and ink jet printer heads whereby excimer laser drilling are key processes $[18,19]$. Our measurements here used an Exitech M8000 mask projection micromachining system incorporating a Lambda Physik LPX201 KrF laser with a beam delivery system consisting of shaping lenses, variable attenuator, fly's eye homogenizer arrays, field lens and a 0.13NA, $\mathrm{x} 5$ demagnification diffraction-limited imaging objective [20]. For our results presented here arrays of $5 \mu \mathrm{m}$ and $50 \mu \mathrm{m}$ wide channels were micromachined in $80 \mu \mathrm{m}$ thick polyimide tape mounted on silicon wafers using range of incident fluences and numbers of pulses. Following sectioning to provide cross-section profiles, ablation depths and entrance wall angles were measured from SEM micrographs.

\section{Laser illumination of microstructures}

It is important to understand how the topology of a microstructure created by successive pulses can modify its illumination characteristics - particularly in cases when high numerical aperture mask imaging or beam focusing lenses are used to define micron-sized features. To illustrate how such effects could modify the topology of the structure produced, we have used the Zemax optical ray tracing code [21] to model the flux distribution at the bottom of simple blind hollow volumes in substrates with polyimide-type optical properties at $248 \mathrm{~nm}$. No paraxial approximations are made in these calculations and ray splitting of the randomly polarized beam at refractive walls provides the correct power sharing between reflected, refracted and transmitted components for all ray angles incident. To study the impact wall reflections have on the illumination characteristics of the microstructure, ray splitting in the code can be disabled which then gives zero power to reflected ray components. A Monte Carlo generation of $10^{6}$ illumination rays is traced through the system. The incident spatial distribution of power, the peak fluence and total energy captured are then calculated on a 100x100 2-D pixel array virtual detector element situated at the floor of the channel. Fluence distributions calculated at the bottom of a tapered channel with and without reflections included in the computations are shown in Fig 1(a).
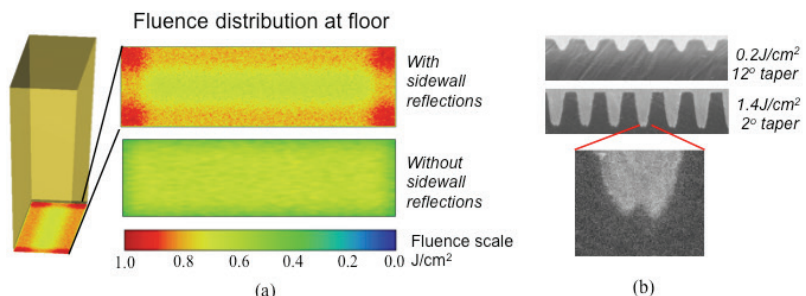

(b)

Fig. 1. (a) Fluence distribution maps calculated at bottom of a $2^{\circ}$ tapered, $12.4 \mu \mathrm{m}$ deep, $5 \times 15 \mu \mathrm{m}^{2}$ channel, when illuminated at a uniform fluence and divergence of $0.65 \mathrm{~J} / \mathrm{cm}^{2}$ and $0.13 \mathrm{NA}$ respectively, with and without reflections at the sidewalls. (b) $5 \mu \mathrm{m}$ wide channels on a $10 \mu \mathrm{m}$ pitch machined in polyimide using $30 \mathrm{KrF}$ laser pulses at $0.2 \mathrm{~J} / \mathrm{cm}^{2} /$ pulse (upper) and $1.4 \mathrm{~J} / \mathrm{cm}^{2} /$ pulse (lower) together with a magnified image of the bottom of a channel.

As can be seen the effect of wall reflections is to redistribute power away from the middle of the channel to its edges and corners, which in this example concentrates the illumination fluence in these regions by up to $35 \%$. This fluence enhancement at the edges and corners will have the effect of increasing the ablation rate in these regions to create a floor with a raised profile. To compare this behavior with experimental observations, in Fig 1(b) we show examples of arrays of channels machined at relatively high and low pulsed laser fluences. While the increase in depth and reduction of taper angle with fluence are clearly evident, in the magnified image in Fig. 1(b) a raised hump in the middle of the channel floor is clearly seen. This island nature to the floor of blind micromachined structures has been observed by others and postulated to be due to diffractive effects at the edges of the illuminated region [22, 23, 24, 25]. However as illustrated in Fig 1(a) the redistribution of power by wall reflections show diffractive effects are not necessary to explain this behavior - a conclusion also reached by the workers in Ref. 11.

To illustrate further the effects a micromachined structure topology can have on its illumination as a result of reflections and refractions at its walls, in Fig. 2 we show calculations of the fall off and redistribution of power with depth for a $30 \times 30 \mu \mathrm{m}^{2}$ rectangular hollow channel with straight walls in a polyimide-like material. The power loss is calculated from the total power received by the detector compared to the power illuminating the surface. The graph shows sidewall reflections reduce the power loss down the channel - in this case by about $5 \%$ at a depth of $20 \mu \mathrm{m}$ rising to $20 \%$ at $100 \mu \mathrm{m}$. Furthermore it can be seen in the fluence maps at the bottom of the figure the power drops off rapidly as refraction through the walls into the substrate 
takes power away from the channel. For example, increasing the depth from $20 \mu \mathrm{m}$ to $50 \mu \mathrm{m}$ more than doubles the overall loss in power.

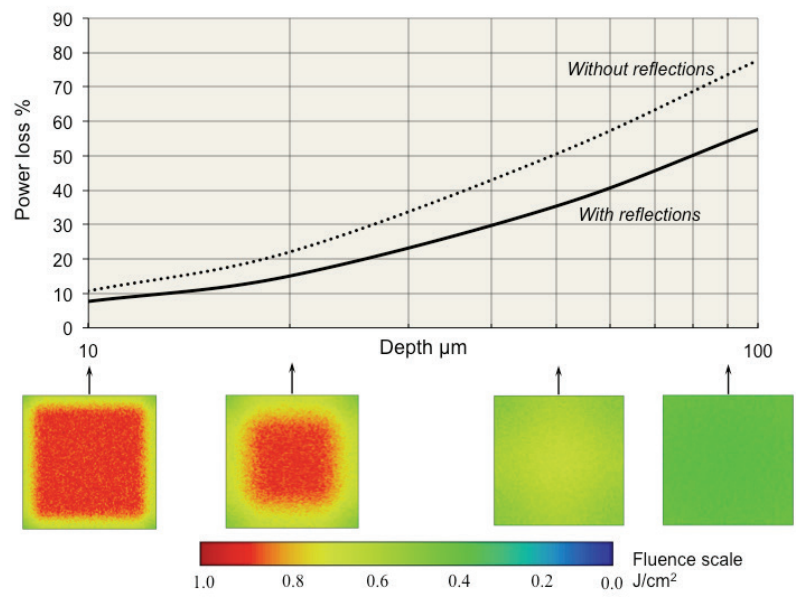

Fig. 2. Power loss and fluence distribution at the bottom of a $30 \times 30 \mu \mathrm{m}^{2}$ channel in polyimide as a function of its depth when illuminated by a uniform beam at a fluence and numerical aperture of $0.89 \mathrm{~J} / \mathrm{cm}^{2}$ and $0.42 \mathrm{NA}$ respectively.

Likewise the simulations in Fig. 3 show increasing the numerical aperture of the illumination of a straightwalled channel also leads to a rapid loss of power down the channel. As the numerical aperture increases, the angle of incidence on the walls decreases so more energy becomes lost by refraction through the sidewalls into the substrate. In this case increasing the illumination from $0.42 \mathrm{NA}$ to $0.7 \mathrm{NA}$ more than doubles the loss of power.

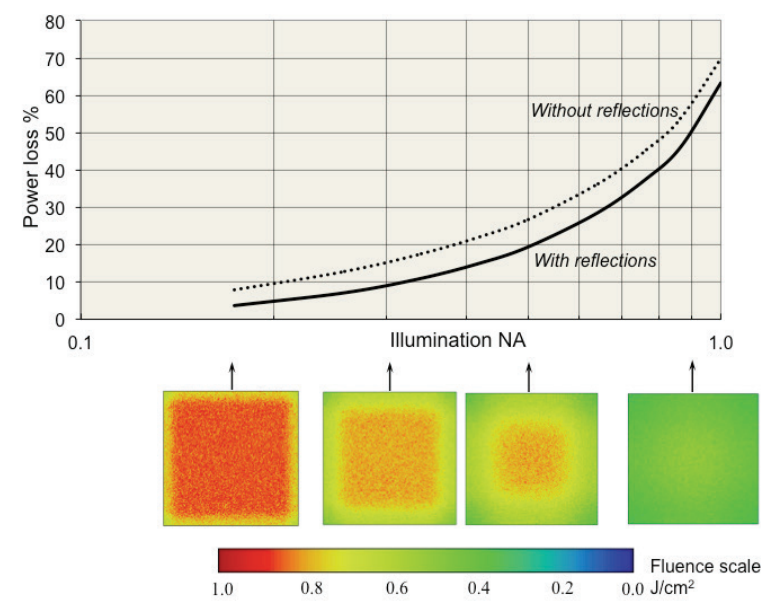

Fig. 3. Power loss and fluence distribution calculations at the bottom of a $30 \times 30 \times 20 \mu \mathrm{m}^{3}$ channel in polyimide with nontapering walls as a function of the numerical aperture of the illumination when illuminated by a uniform beam at a fluence of $0.78 \mathrm{~J} / \mathrm{cm}^{2}$.

The influence of tapering walls has a pronounced effect on the illumination properties. In Fig. 4 we show calculations of the power loss and fluence distribution at a depth of $20 \mu \mathrm{m}$ for a $30 \times 30 \mu \mathrm{m}^{2}$ rectangular entrance light guiding hollow volume as a function of its positively tapering wall angle. The graph shows sidewall reflections reduce the power loss down the channel - in this case uniformly by about $20 \%$. In addition the fluence distribution maps at the bottom of the figure show as the floor area is reduced due to the increasing wall taper angle, power becomes concentrated and redistributed from the middle of the beam to the edges and corners of the channel - similar to that shown in Fig. 1(a). In this example at a $10^{\circ}$ wall angle, the average fluence at the bottom of the channel is $20 \%$ higher than that at the surface, and at the edges of the floor is $30 \%$ higher than in the middle.

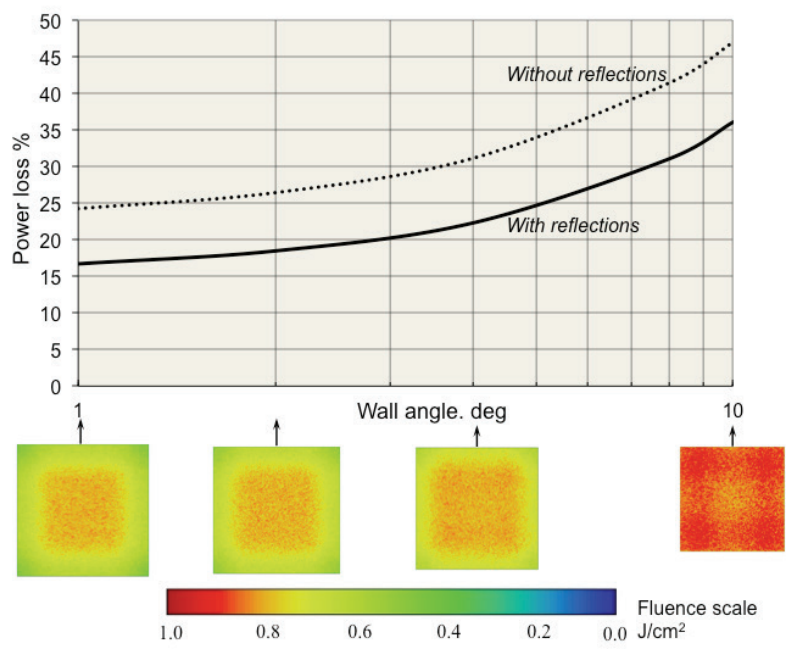

Fig. 4. Power loss and fluence distribution calculations at the bottom of a $30 \times 30 \times 20 \mu \mathrm{m}^{3}$ channel in polyimide as a function of the taper angle of the walls between $1^{\circ}-10^{\circ}$ when illuminated

by a uniform beam at a fluence and numerical aperture of $0.78 \mathrm{~J} / \mathrm{cm}^{2}$ and $0.42 \mathrm{NA}$ respectively.

From the simulations in Figs. 1-4 we conclude positively tapered walls produce several beneficial effects in aiding the creation of deep micromachined structures. By concentrating the beam in waveguiding reflections and redistributing power to the edges, tapering walls help overcome the refractive losses in fluence experienced at increasing depths and in microstructures illuminated at high numerical apertures.

\section{Laser ablation of microstructures pulse-by- pulse}

We also use the Zemax code [21] to model the pulse-bypulse creation of microstructures using the spatial profile of the energy incident on and absorbed in a substrate. For each laser pulse $10^{5}-10^{6}$ rays in a Monte Carlo generation of illumination rays are traced from the laser through the beam delivery system and onto the substrate. We use the code to model the substrate as a 'detector volume' that is subdivided into 3-D voxels (volume pixels) for which the incident and absorbed flux on each can be calculated up to a maximum number of $10^{7}$. Photon absorption in the substrate is assumed to be according to Beer's Law placing the computations in the 'photochemical' or 'cold ablation' regime.

\subsection{Photon absorption and the ablation threshold} Two important parameters that determine the ablation characteristics in the photochemical regime are the threshold ablation fluence $E_{T}$ given approximately by [5]: 


$$
E_{T}=\frac{m h v}{\alpha \eta(1-R)}
$$

and the depth ablated per pulse $\Delta z$ given by $[26,27]$ :

$$
\Lambda z=\frac{1}{\alpha} \ln \left(\frac{E}{E_{T}}\right)
$$

where $E$ is the exposure fluence, $\mathrm{m}$ the number of molecular bonds which must be broken per unit volume, $\eta$ the quantum yield for chain scission, $h v$ the photon energy assumed to exceed the bond energy of the polymer chain and $R$ the surface reflection loss. Eq. 1 can be rewritten:

$E_{T} / L=C$

where $L=1 / \alpha$ is the photon absorption depth and $C$ a constant for a given wavelength and material. Expressing $\Delta z$ as $\Delta z^{\prime}$ in terms of units of $L$, and $E$ as $E^{\prime}$ in units of $E_{T}$, Eq. 2 becomes:

$$
\Delta z^{\prime}=\ln E^{\prime}
$$

Note from Eqs. $3 \& 4$, the relative ablated depth $\Delta z^{\prime}$ remains unchanged when $E, E_{T}$ and $L$ are all scaled together in the same ratio. This property becomes useful for some computations in order to simplify some of the optical components that would otherwise be needed when using the very small values of $L$ and short photon propagation distances typically encountered in excimer laser ablation of polymers.

\subsection{Substrate voxels}

Referring to Fig. 5, taking a j-th ray incident on a 3-D voxel in the substrate travelling at an angle $\Theta_{j}=\left(\theta_{x}, \theta_{y}\right.$, $\theta_{z}$ ) with an incident flux $I_{j}\left(A_{j}, \Theta_{j}\right)$ through a path length $\ell_{j}$ $=\left(\ell_{x}, \ell_{y}, \ell_{z}\right)$, then, for a total of p-rays arriving at the voxel, the total incident, absorbed and transmitted fluxes $F_{I}, F_{A}, F_{T}$ associated with it are:

$$
F_{I}=\sum_{j=1}^{p} I_{j} \quad F_{A}=\sum_{j=1}^{p} I_{j} e^{-\alpha \ell_{j}} \quad F_{T}=\sum_{J=1}^{p} I_{j}\left(1-e^{-\alpha \ell_{j}}\right)
$$

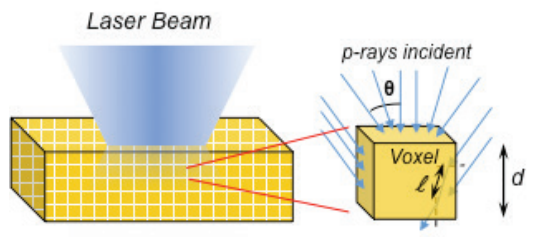

Fig. 5. Illustration of rays incident on a substrate voxel of cubic dimensions $d$.

To calculate the topology of the structure micromachined by each pulse, each substrate voxel receiving an incident fluence above the threshold for ablation is assigned a hollow volume to create a new overall optical volume ready for illumination by the next pulse. Computations of the evolution of the ablated volume on a pulse-by-pulse basis are automated by a macro performing repetitive calculations of the process.

Since subdividing the substrate into voxels could have an influence on the calculation of the quantity and topology of material removed, it is important the voxel dimensions chosen do not impact the calculation. To evaluate the voxel size regime where its dimensions are unimportant, we consider the simple model of subsurface 2-D pixels illuminated by a collimated beam from above at a fluence exceeding the threshold for ablation. Then using Beer's law the number of pixels ablated $q$ is:

$$
q=1+\frac{1}{d^{\prime}} \ln \left(E^{\prime}\right)
$$

rounded down to the nearest integer, and where the pixel dimension $d^{\prime}$ is in units of $L$. The relative linear depth ablated is then given by:

$$
\Delta z^{\prime}=q d^{\prime}
$$

Since only an integral number of pixels can be ablated, the rounding down to the nearest integer value in Eq. 6 is an important consequence of pixelating the substrate. Straightforward substitution of Eq. 6 into Eq. 7 without this rounding produces an incorrect result - similar to Eq. 4 but with an additional term $d^{\prime}$.

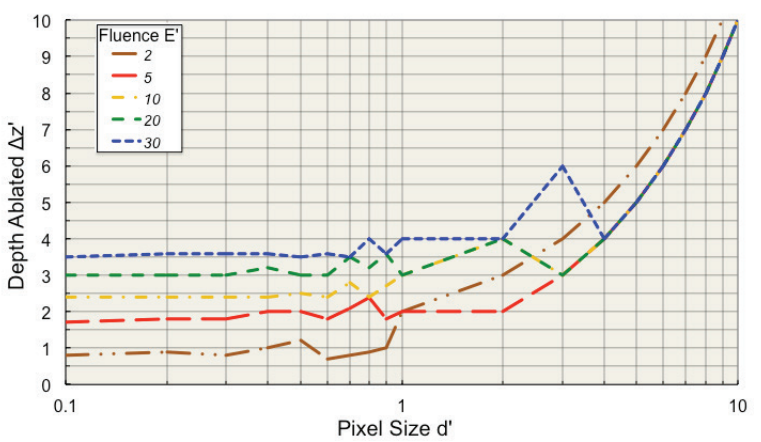

Fig. 6. Plot of Eq. 7 of relative depth ablated $\Delta z^{\prime}$ versus relative pixel size $d^{\prime}$.

The plots in Fig. 6 show that for relative fluences $E^{\prime}=1$ -30 , the relative depth ablated is insensitive to the pixel size chosen for values of $d^{\prime} \leq 3$. To achieve calculations with the highest spatial resolution without using the parameter scaling discussed in Section 3.1, voxel dimensions between $d^{\prime}=0.5-3$ were chosen in the results presented in later sections.

\subsection{Model validation}

For polyimide irradiated with $\mathrm{KrF}$ laser pulses, the photochemical model works well for fluences in the range $E \leq 0.6 \mathrm{~J} / \mathrm{cm}^{2} /$ pulse or $E^{\prime} \leq 30[7,15]$. Many excimer laser ablation studies in this fluence regime have been reported in the literature for this material. For our calculations at $248 \mathrm{~nm}$, we use values for the refractive index $n=1.79$; the absorption coefficient $\alpha=2.8$ 
$\mathrm{x} 10^{5} \mathrm{~cm}^{-1}$; the photon absorption depth $L=36 \mathrm{~nm}$ and threshold fluence for ablation $E_{T}=19 \mathrm{~mJ} / \mathrm{cm}^{2} /$ pulse $[28,29]$. Validation of the ablation model was made by comparing calculations of the depth ablated and wall taper angle with experimental measurements made by ourselves and others using this material. While experimental results for $248 \mathrm{~nm}$ laser ablation of polyimide are used to validate the model, it can be applied much more widely to pulsed laser ablation of many other material types at wavelengths, pulse durations and fluences for which the ablated depth is observed to have a Beer's Law type of logarithmic dependence on incident fluence (Eq. 2). For example, ablation rates of polyethylene terephthalate (PET), polymethyl methacrylate (PMMA) and polycarbonate (PC) at short wavelengths show a similar behavior in the low fluence 'photochemical' regime typically for values of $\mathrm{E}$ up to $\mathrm{x} 10-30 \mathrm{E}_{\mathrm{T}}[7,9,12,26,27]$. Indeed the ablation rate of PET by a pulsed $\mathrm{CO}_{2}$ laser at the infrared wavelength of $9.2 \mu \mathrm{m}$ also shows a logarithmic dependency on fluence [30]. However the model will be less applicable for those polymers at wavelengths in fluence regimes that show a behavior departing from this relationship. Such departures can be caused by incubation when, for example, PMMA being a weak absorber at $248 \mathrm{~nm}$ is irradiated just above its ablation threshold at this wavelength [31], or more generally at high UV laser fluences $\gtrsim 1 \mathrm{~J} / \mathrm{cm}^{2}$ when effects such as thermal decomposition, ablation product and plasma screening become evident [32-34]. Even in such cases it may be possible to use a modification to the room temperature absorption coefficient $\alpha$ to take account of effects such as an increase in material absorptivity with temperature [26] and screening of the tail of the pulse by the ablation plume [34].

However in cases where the ablation process does not follow a Beer's Law type of behavior this model will not be applicable - for example, when long duration laser pulses are used to create material removal by thermal processes; when thermal conductors like metals in bulk or thin film forms are ablated; at the high peak intensities typical encountered at the focus of ultrashort laser pulses which cause nonlinear optical absorption of photons to act as a precursor to the ablative removal of material.

\subsubsection{Ablation rate}

Calculations of the ablation depth were made using a single pulse of a $1 \mu \mathrm{m}$ square unfocussed parallel beam with a uniform intensity distribution irradiating a polyimide sample. Using $10^{5}$ analysis rays/pulse and cubic voxels with dimensions $d=18 \mathrm{~nm}\left(d^{\prime}=0.5\right)$, gave 8.3rays/pulse on average illuminating each surface voxel. The results of these calculations are shown in Fig. 7 compared with the single pulse ablation rate measured by other workers and ourselves in the photochemical and medium fluence regimes. Given the relatively large uncertainty most workers face in making accurate absolute incident beam energy and area measurements, there is relatively good agreement between the modeling calculations and experimental data.

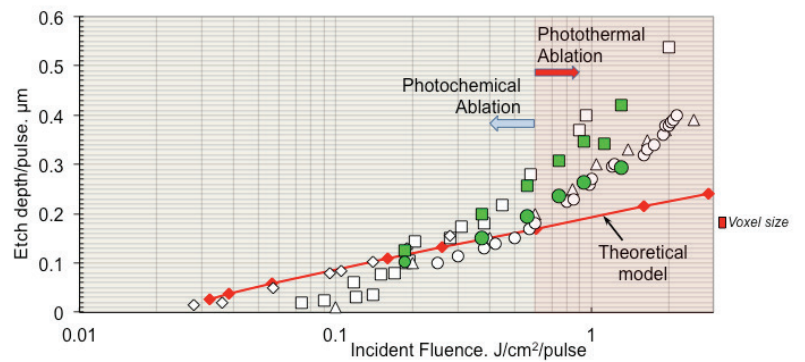

Fig. 7. $\mathrm{KrF}$ laser ablation rate for polyimide as a function of single laser pulse fluence. Measurements: $\square$ Ref 12; $\diamond$ Ref 13; $\Delta \operatorname{Ref} 13 ; \bigcirc \operatorname{Ref} 14$; This work: $5 \mu \mathrm{m}$ channels, - $50 \mu \mathrm{m}$ channels. Calculations: $\triangle$ Model

Our experimental data in Fig. 7 shows the ablation rate for $5 \mu \mathrm{m}$ wide channels was $20-30 \%$ larger than for $50 \mu \mathrm{m}$ wide channels. Other workers have also noticed an enhancement to the ablation rate at smaller feature sizes $[11,23,35]$. As discussed in Section 2, this behavior is most likely due to the greater influence wall reflections have on increasing the fluence incident at the bottom of narrower channels. The experimental data shows that going from the photochemical to the photothermal regime at fluences $E>0.6 \mathrm{~J} / \mathrm{cm}^{2} /$ pulse, the ablation rate increases above that predicted from Beer's law as molecular bond scission by thermal processes starts to take effect. In the thermal regime, rather than Eq. 2, the depth ablated per pulse $\Delta z$ can be described by the equation [36]:

$$
\Delta z=A \exp \left\lfloor-\frac{\varepsilon}{\alpha E} \ln \left(\frac{E}{E_{T}}\right)\right\rfloor
$$

where $A$ is a constant and $\varepsilon$ the activation energy of the material at the laser wavelength.

\subsubsection{Wall taper}

There have been many suggestions put forward to explain the initial tapering of wall angles observed when ablating microstructures in polymers with excimer laser radiation. Among these are diffraction of light at the edges of features [22-25,35], blurring of edges due to optical imperfections in imaging systems, mechanical jitter and thermal effects [11], the existence of a critical angle of incidence due to an ablation threshold [17], wall reflections and details of the optical imaging system particularly the numerical aperture (NA) of illumination [8]. The agreement between the experimental results and theory by the workers in Ref. 8 indicates the taper produced in imaging systems is a consequence of offaxis mask illumination characteristics of beam homogenizers and the imaging objective NA. Our raytracing model, which did not include diffraction effects, confirms this. Also for contact masks illuminated by flood exposure from a focused beam it has been suggested diffraction at mask edges is again responsible for tapering walls [35]. We will see in Sect. 3.4 diffraction effects are not necessary to explain this behavior either. 
Calculations of the changes in the ablation wall angle with fluence were made using 20 pulses of a Gaussian beam focused on the surface of polyimide. In this case, to simplify the focusing optics to one of only a singlet lens, computations were carried out by scaling $E, E_{T}, L$ and dimensions together by the same factor - in this case 27,233x. A $70 \mathrm{~mm}$ focal length lens was used to focus a $45 \mathrm{~mm}$ diameter Gaussian beam to a $9 \mathrm{~mm}$ diameter spot on the substrate surface. Using a total of $10^{4}$ analysis rays/pulse giving 3.85 rays/surface voxel/pulse on average, calculations of the entry wall angle as a function of the incident laser fluence are shown in Fig. 8 together with measurements made by ourselves and others.

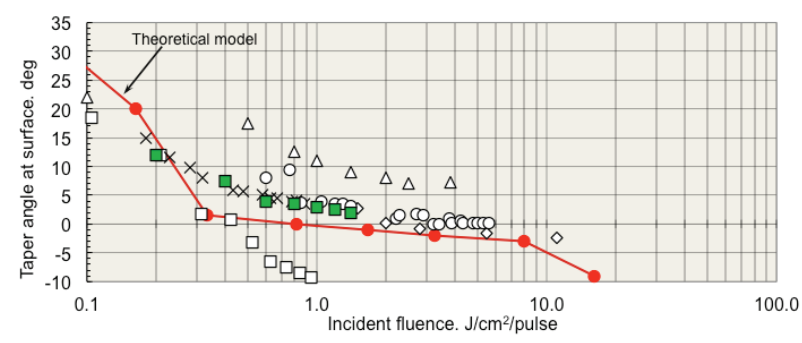

Fig. 8. $\mathrm{KrF}$ laser ablated wall taper angle for polyimide as a function of single laser pulse fluence.

Measurements: $\times$ Ref 11, 0.033NA, 179pulses, $64 \mu \mathrm{m}$ holes; $\bigcirc$ Ref 15, 0.2NA, 200pulses, $46 \mu \mathrm{m}$ trenches; $\square$ Ref 16, 0.3NA, $\sim 300$ pulses, $50 \mu \mathrm{m}$ holes; $\triangle$ and $\diamond$ Ref $17,0.02 \mathrm{NA}$ and $0.16 \mathrm{NA}$ respectively, $60 \mu \mathrm{m}$ holes; $\square$ This work, $0.13 \mathrm{NA}, 30$ pulses,

$5 \mu \mathrm{m}$ trenches; Calculations: $\bullet$ Model, 0.32NA, 20pulses

There is broad agreement between the calculations and experimental data, particularly so given the wide range of very different experimental conditions (as given in the figure caption) all of which are known to affect the taper angle produced - focused or imaged beams, illumination NA, number of pulses, ablation hole/channel size, etc. This comparison too is really only appropriate to make in the photochemical regime for incident fluences $E \leq$ $0.6 \mathrm{~J} / \mathrm{cm}^{2} /$ pulse where the model is valid.

\subsection{Ablation pulse-by-pulse}

For many industrial applications of excimer laser ablation in e.g. drilling microvia holes and ink jet printer nozzles, it is important to be able to predict both the shape of the hole produced and the most efficient use of photons to remove a requisite amount of material. Many parameters can contribute to the most efficient machining strategy: pulse energy fluence, number of pulses, beam profile, mask/aperture shape, beam delivery system, imaging/focusing optics and position of the focal/image plane.

\subsubsection{Microholes ablated by a focused beam}

A simple example shown in Fig. 9 uses the scaling arguments mentioned previously to calculate the development of a $9 \mathrm{~mm}$ diameter hole drilled by a sequence of pulses focused inside a $48 \mathrm{~mm}$ cube of material. As for the taper angle calculations in Fig. 8, $E$, $E_{T}, L$ and dimensions have all been scaled by $\mathrm{x} 27,233$ to simulate the case of $\mathrm{KrF}$ laser ablation of polyimide. It can be seen from this figure that as the hole becomes deeper as more pulses remove material the focus moves upwards in the sample as the lower index air structure is created. As the hole approaches the focal position a positively tapered structure mimics the beam profile of the focused beam. Further pulses remove material from the walls to open out the structure. Beyond focus additional pulses guided by reflections in the structure remove material from the walls producing little or even a small negative taper in creating a through hole.

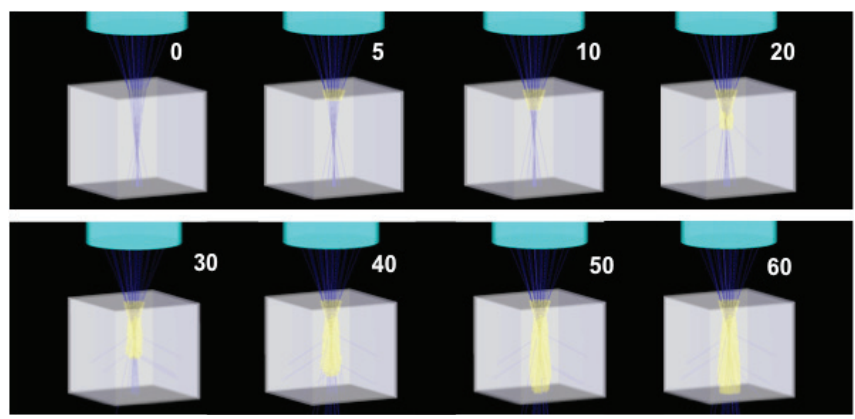

Fig 9. Scaled calculation simulating $\mathrm{KrF}$ laser ablation of polyimide as a function of number of pulses as shown in each picture. $45 \mathrm{~L}$ diameter Gaussian beam brought to a focus inside a $48 \mathrm{~L}$ cube using a $70 \mathrm{~L}$ focal length singlet lens. At the surface $E^{\prime}=8\left(0.16 \mathrm{~J} / \mathrm{cm}^{2} /\right.$ pulse equivalent $) . d^{\prime}=1$ cubic voxels and $10^{4}$ analysis rays/pulse. The $27,233 \mathrm{x}$ scaled photon absorption depth $L=1 \mathrm{~mm}$. Rays shown are for illustrative purposes only.

To study the behaviour of the hole shape and efficiency for removing material, in Fig. 10 we show similar calculations for the behaviour when the surface of a substrate is placed before, at and after the focus of a Gaussian beam using the same numbers of pulses for the calculations. In Fig. 10(a) as in Fig 9 with the focus inside the material, the hole mimics the intensity profile of the focused beam towards focus producing a positively tapered hole at the surface. With a characteristic countersunk shape, beyond focus it straightens out and even produces a small negative taper. Contrast this behaviour to Fig. 10(b) when focusing on the surface produces a much smaller straight through hole similar in diameter to the dimensions beyond focus in Fig. 10(a). Since about half as much material is removed in Fig. 10(b) for the same laser beam parameters as in Fig. 10(a), focusing below the surface can be regarded as a more efficient drilling strategy.

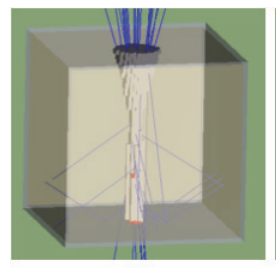

(a)

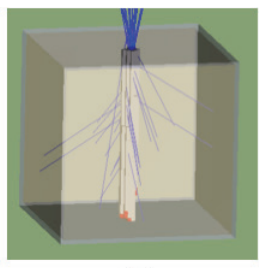

(b)

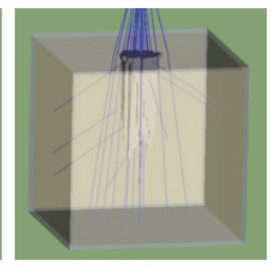

(c)
Fig 10. Scaled calculation simulating $\mathrm{KrF}$ laser ablation of polyimide using 22 pulses and a $45 \mathrm{~L}$ diameter Gaussian beam. (a) Substrate positioned inside the focus. At the surface $E^{\prime}=3$ $\left(0.06 \mathrm{~J} / \mathrm{cm}^{2} /\right.$ pulse equivalent); (b) Substrate positioned with the focus at the surface. At the surface $E^{\prime}=83.3\left(1.7 \mathrm{~J} / \mathrm{cm}^{2} / \mathrm{pulse}\right.$ equivalent); (c) Substrate positioned beyond the focus with a surface fluence similar to (a). $d^{\prime}=1$ cubic voxels and $10^{4}$ analysis rays/pulse. The $27,233 x$ scaled photon absorption depth $L=1 \mathrm{~mm}$. Rays shown are for illustrative purposes only. 
When wall reflections for the 'at focus' calculation are turned off, the straight shape of the hole remains largely unchanged, although about 30\% more pulses would be required to drill the same depth. For completeness, in Fig 10 (c) we show the case for the substrate placed beyond focus. In this case an entrance hole with a positive taper is also produced as a result of the beam fluence reducing with propagation distance eventually leading to cessation of material removal and a blind hole. Similar shaped holes are calculated when a hard aperture mask is placed on the surface. The behaviour in Figs. 10(a)-(c) is consistent with the initially positively tapering holes observed in experiments when using a focused beam to flood expose mask apertures placed in contact with the substrate [29]. We conclude that for dimensions several times large than the wavelength of the laser source, edge diffraction is not necessary to explain the initiation of a positively tapering wall shape to an ablated hole. Without a focusing lens to concentrate the beam, as one might expect, our calculations showed a beam at normal incidence with zero divergence and uniform intensity distribution produced structures having straight vertical walls.

\subsubsection{Microstructures ablated by mask projection}

Fig. 11(a) shows a typical excimer laser beam delivery system [37,38] which we have used for illustrative calculations of ablated microstructures created by projection of a mask pattern. A x36, 0.5NA Schwarzschild reflective objective is used to demagnify images of object mask patterns onto the substrate. Because the small mirror in these objectives obscures the centre of the image field, optimal imaging is achieved using off-axis illumination of the object. In this example this is achieved using a double Fresnel biprism (quadprism) to split the beam into four and then recombine each back together at the mask plane.

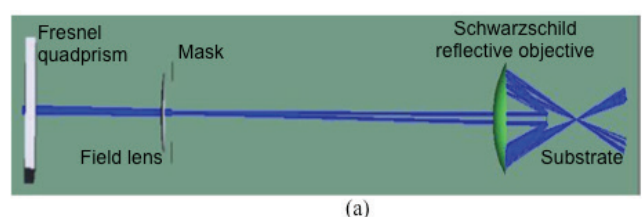

(a)

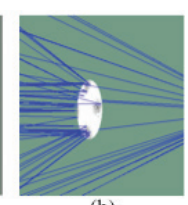

(b)
Fig 11. (a) Excimer laser beam delivery system comprising: A Fresnel quadprism for providing off-axis illumination of a mask; field lens; transmission mask; a x36, 0.5NA

Schwarzschild reflective imaging objective; (b) Rays and intensity profile at the entrance pupil on the small primary Schwarzschild mirror.

Because this component also folds each beamlet back on itself there is the added benefit of the beam profile being homogenized at the mask plane [14]. The angles of the facets for the quadprism were chosen to give a coherence factor $\sigma=0.7$ - the ratio of the illumination to the imaging NA, which is optimal for providing the best imaging resolution with off-axis illumination. Finally a field lens near the mask is used to focus the radiation into the entrance pupil of the objective, which in this case is located close to the small primary mirror. Fig. 11(b) shows the rays incident and intensity distribution on this mirror with the four focused beamlets.
A typical $\mathrm{KrF}$ laser beam - randomly polarized, low spatial and temporal coherence with a divergence of $3 \mathrm{mrad}$ is used to illuminate the system. More complex systems can be used with the model e.g. laser sources producing highly temporally and spatially coherent polarized beams with beam delivery systems having components such as fly's eye array homogenizers, multielement refractive, wide image field objectives and phase shifting masks.

Fig. 12(a) shows a sequence of nominally $1 \mu \mathrm{m}$ diameter blind microhole shapes calculated at increasing fluences when a circular aperture is illuminated and imaged on a polyimide substrate using the beam delivery system in Fig 11(a).

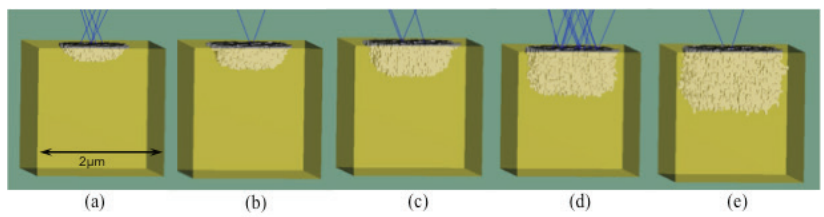

Fig 12. $1 \mu \mathrm{m}$ diameter tapered blind microholes created after 5 $\mathrm{KrF}$ laser pulses incident on a $2 \mu \mathrm{m}$ polyimide cube. $36 \mathrm{~nm}\left(d^{\prime}\right.$ $=1$ ) cubic voxels and $10^{5}$ analysis rays/pulse. Incident fluences: (a) $0.046 \mathrm{~J} / \mathrm{cm}^{2} /$ pulse; (b) $0.085 \mathrm{~J} / \mathrm{cm}^{2} /$ pulse; (c)

$0.185 \mathrm{~J} / \mathrm{cm}^{2} /$ pulse; (d) $1.07 \mathrm{~J} / \mathrm{cm}^{2} /$ pulse; (e) $7.46 \mathrm{~J} / \mathrm{cm}^{2} /$ pulse. Rays shown are for illustrative purposes only.

It can be seen that as the incident fluence increases, the taper angle of the wall becomes less. Even though the fluences in Fig 12(d) and (e) are beyond the purely 'photochemical' regime, it is apparent the hole shapes begin to exhibit negative taper and undercut akin to the results shown in Fig. 8.

This behavior is further illustrated by the calculations shown in Fig. 13(a) that use the same beam delivery system but a different mask feature simulating micromachining a $6 \times 2 \mu \mathrm{m}^{2}$ elongated channel. Because of the off-axis illumination of the mask, the channel begins to bifurcate from its single channel entrance at the surface. We compare this behavior to the experimental result [16] of two undercut rectangular channels machined through the backside of a polyimide sheet using a similar beam delivery system to that used for our calculations - with the exception of using a biprism instead of a quadprism illuminator.
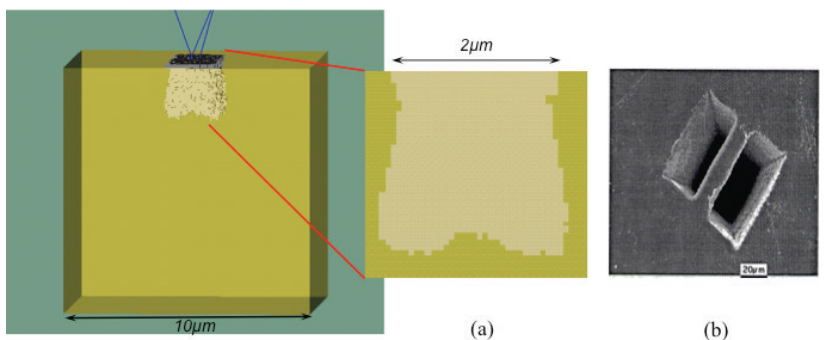

Fig 13. (a) $6 \times 2 \mu \mathrm{m}^{2}$ channel in $10 \mu \mathrm{m}$ polyimide cube using the beam delivery system in Fig.12(a) with $30 \mathrm{KrF}$ laser pulses at

$1.1 \mathrm{~J} / \mathrm{cm}^{2} /$ pulse. $72 \mathrm{~nm}\left(d^{\prime}=2\right)$ cubic voxels and $10^{5}$ analysis rays/pulse. A total of 55,314 voxels were removed equivalent to $20.6 \mathrm{~m}^{3}$ of material. (b) Exit side of a polyimide sheet in which a bifurcated channel has been micromachined using a $\mathrm{KrF}$ laser and a similar beam delivery system [16]. 
With this technique the authors of Ref. 16 noted a wide variety of differently shaped multi-channeled structures can be machined and, by increasing the number of offaxis beam components illuminating the mask, the number of channels can be increased.

To illustrate further the behavior that an optical beam delivery system can have in determining the shape of an ablated microstructure as well as the ability to model substrates with material interfaces, in Fig. 14 we show the calculations produced by the delivery system in Fig. 11(a) when the image of a mask containing a small spoked wheel structure is used to ablate a thin film of polyimide mounted on a silicon substrate.

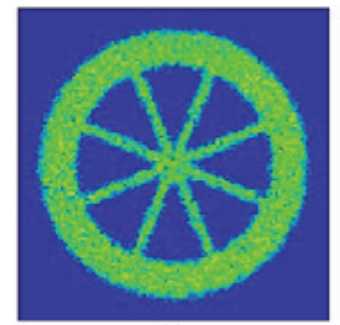

(a)

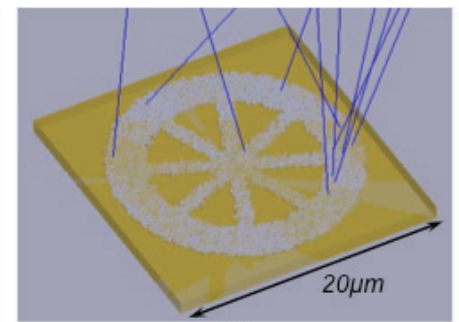

(b)
Fig 14. (a) The $\mathrm{KrF}$ laser illumination intensity profile of the mask image of a wheel on the surface of the substrate. Imaged wheel diameter $=17.2 \mu \mathrm{m}$, spoke width $=0.6 \mu \mathrm{m}$; (b) Structure ablated by 4 pulses at $0.67 \mathrm{~J} / \mathrm{cm}^{2} /$ pulse in $1 \mu \mathrm{m}$ thick polyimide film on silicon. $180 \times 180 \times 36 \mathrm{~nm}^{3}(5 L \times 5 L \times L)$ voxels and $2.5 \times 10^{4}$ analysis rays/pulse.

In this case with a mask wheel diameter $=620 \mu \mathrm{m}$ and spoke width $=19 \mu \mathrm{m}$, the $\mathrm{x} 36$ reduced image produced by the Schwarzschild objective is faithfully reproduced in the ablated profile through the polyimide film (Fig. 14(b)). In particular the $0.6 \mu \mathrm{m}$ wide spokes are fully resolved and replicated in the film.

In contrast, calculations using a mask pattern half the size of that used in Fig. 14 are shown in Fig. 15. In this case the spokes of the wheel in the image should be $0.3 \mu \mathrm{m}$ wide. However as seen in Fig. 15(a) and (b) they remain $0.6 \mu \mathrm{m}$ wide. If the objective used for imaging the mask had true diffraction-limited imaging performance at its $0.5 \mathrm{NA}$ design it should be capable of fully resolving $0.3 \mu \mathrm{m}$ wide features.

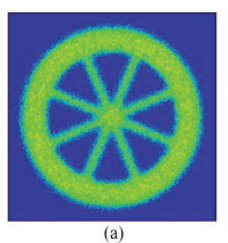

(a)

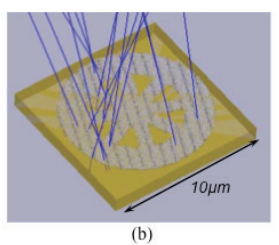

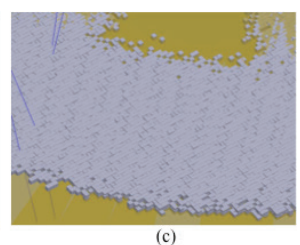

Fig 15. (a) As Fig. 14 except for a wheel mask half the size. (b) Structure ablated by a single pulse at $1.57 \mathrm{~J} / \mathrm{cm}^{2} /$ pulse in $1 \mu \mathrm{m}$ thick polyimide film on silicon. $0.6 \mu \mathrm{m}$ spoke width remains unchanged from Fig. 14(b) due to exceeding the resolution limit of the imaging objective (c) $x 8$ magnified view of the wheel rim region showing $36 \mathrm{~nm}^{3}$ voxels removed. $36 \mathrm{~nm}\left(d^{\prime}=1\right)$ cubic voxels and $10^{6}$ analysis rays removed 228,743 voxels over the entire $8.6 \mu \mathrm{m}$ diameter structure
However the optical aberrations inherent to this type of objective make it incapable of doing so. While the twomirror finite-conjugate modified Schwarzschild design used here is achromatic and free from third-order spherical, coma and astigmatism, it still has residual distortion, field curvature and higher-order aberrations which prevent diffraction-limited imaging performance at large numerical apertures - as is apparent from the simulations shown in Fig. 15(a) and (b).

\section{Summary and Conclusions}

From only a priori knowledge of the optical parameters of the laser beam delivery system together with the substrate material properties, a ray-tracing model capable of predicting the topology of micro/nanostructures machined by pulsed laser ablation has been developed. The model includes secondary illumination effects produced by the microstructure created by successive pulses (wall reflections, refraction, wave guiding, shadowing, etc.) as well as the complete optical properties of the beam delivery system. Good agreement is obtained between the model predictions and experimental results in terms of the predicted depth ablated per pulse and the vertical wall taper angle of channels and holes. The model has been used to predict ablated profiles of holes and to indicate the most efficient drilling strategy in terms of material removal rates. The model also shows diffraction effects are not required to explain tapering vertical walls observed when ablating microstructures. Finally the model has been used to demonstrate aberrations in an optical imaging system that limit the creation of submicron features in a microstructure.

We have concentrated on applying the model to ablative microstructuring with pulsed excimer lasers together with focused and mask imaging beam delivery systems. However, provided photons are absorbed linearly in a substrate according to Beer's law with negligible thermal diffusion effects, it is equally applicable to using other types of short pulsed laser sources and beam delivery systems with imaged or focused beams. However the relatively large material absorption properties encountered in nanosecond excimer laser pulse ablation of polymers make it particularly useful for modeling the fabrication of ablated nanostructures having feature sizes $10-100$ times larger than the photon absorption depth in the material.

\section{References}

[1] Y Kawamura, K Toyoda and S Namba, Appl. Phys. Letts, 40, 374, (1982)

[2] R Srinivasan and V Mayne-Banton, Appl. Phys. Letts., 41, 576, (1982)

[3] G M Davis, A F Gibson, M C Gower, R A Lawes and R A Moody, Microcircuit Engineering '83, Eds. H Ahmed, J R A Cleaver and G A C Jones, (Academic Press, London, 1983), P191

[4] C Brown, B Child, S Hallewell, K Hollingworth, N Prior and P Rumsby, Central Laser Facility Annual Report RAL 83-043, (SERC Rutherford Appleton 
Laboratory, 1983). P.1.24. Available at www.clf.rl.ac.uk/resources/pdf/ar82-83.pdf

[5] P E Dyer, 'Laser Ablation Of Polymers' In Photochemical Processing Of Electronic Materials, Eds. I.W. Boyd and R.B. Jackman, (Academic Press, London, 1992)

[6] P E Dyer, Appl. Phys A77, 167 (2003)

[7] V Srinivasan, M A Smrtic and S V Babu, J Appl. Phys. 59, 3861 (1986)

[8] C Paterson, A S Holmes and R W Smith, J Appl. Phys. 86, 6538 (1999)

[9] J E A Pedder and A S Holmes, Proc. $6^{\text {th }}$ International Symposium on Laser Precision Microfabrication (Japan Laser Processing Society, Williamsburg, VA), 215 (2005)

[10] J E A Pedder and A S Holmes, Proc SPIE 6106, 286 (2006)

[11] T W Hodapp and P R Fleming, J Appl. Phys. 84, 577 (1998)

[12] R Srinivasan and B Braren, J. Poly. Sci. 22, 2601 (1984)

[13] J H Brannon, J P Lankard, A I Baise, F Burns and J Kaufman, J. Appl. Phys. 58, 2036 (1985)

[14] M C Gower, In Laser Processing In Manufacturing, Eds. R C Crafer and P J Oakley, (Chapman \& Hall, London, 1993), Chapters 8 and 9, p163 and p189.

[15] C-R Yang, Y S Hsieh, G-Y Hwang and Y-D Lee, J. Micromech. Microeng., 14, 480 (2004)

[16] E C Harvey, P T Rumsby, M C Gower and J L Remnant, Proc SPIE 2639, 266 (1995)

[17] H-J Kahlert, U Sarbach, B Burghardt and B Klimt, Proc SPIE, 1835, 110 (1992)

[18] J R Lankard and G E Wolbold, Appl. Phys. A54, 355 (1992)

[19] J M Mrvos and A Murthy, 'Recent Progress In Ink Jet Technologies II’, Ed. E. Hanson, The Society for Imaging Science and Technology (IS\&T), Chapter 2, 66 (1999)

[20] M C Gower, Handbook Of Laser Technology And Applications, Vol III, Eds. C E Webb and J D C Jones, (IoP Publishing, Bristol, 2003), Chapter D1.6, P1661

[21] Radiant Zemax LLC, www.zemax.com

[22] P E Dyer, S D Jenkins and J Sidhu, Appl. Phys. Letts. 49, 453 (1986)

[23] M Eyett and D Bäuerle, Appl. Phys. Letts. 51, 2054 (1987)

[24] J H Brannon, J Vac. Sci. Technol. B7, 1064 (1989)

[25] M Tabat, T R O'Keeffe and W Ho, Proc SPIE 1835, 144 (1992)

[26] J E Andrew, P E Dyer, D Forster and P H Key, Appl. Phys. Lett. 43, 717, (1983)

[27] H H G Jellnick and R Srinivasan, J. Phys. Chem. 88, 3048 (1984)

[28] E Arakawa, M Williams, J Ashley and L Painter, J. Appl. Phys. 52, 3579 (1981)

[29] S Küper, J Brannon and K Brannon, Appl. Phys. A56, 43 (1993)

[30] P. E. Dyer, G. A. Oldershaw, and J. Sidhu, Appl. Phys. B 48, 489 (1989).
[31] S. Küper and M. Stüke, Appl. Phys. A 49, 211 (1989).

[32] E. Sutcliffe and R. Srinivasan, J. Appl. Phys. 60, 3315 (1986).

[33] G. D. Mahan, H. S. Cole, Y. S. Liu, and H. R. Philipp, Appl. Phys. Lett. 53, 2377 (1988).

[34] K. Schildbach, Proc. SPIE 1279, 60 (1990).

[35] B Braren and R Srinivasan, J. Vac. Sci. Tech. B3, 913 (1985)

[36] R Srinivasan, B Braren and R W Dreyfus, J. Appl. Phys. 61, 372 (1986)

[37] P T Rumsby and M C Gower, Proc SPIE 1598, 36 (1991)

[38] E C Harvey, P T Rumsby and M C Gower, Laser Ablation Of Electronic Materials, Eds. E Forgassy And S Lazare, (North-Holland, 1992), P255

(Received: June 09, 2012, Accepted: January 05, 2013) 